\title{
NEURODERECHO: ADAPTABILIDAD DE LA NORMATIVA DE DERECHOS HUMANOS CON RELACIÓN A LAS NUEVAS NEUROTECNOLOGÍAS Y PROPUESTAS PARA SU AMPLIACIÓN ${ }^{1}$
}

\section{NEUROLAW: ADAPTABILITY OF HUMAN RIGHTS REGULATIONS IN RELATION TO NEW NEUROTECHNOLOGIES AND PROPOSALS FOR THEIR EXPANSION}

\section{Nicolás Ezequiel Llamas ${ }^{a}$ y José Ángel Marinaro ${ }^{b^{*}}$}

Fechas de recepción y aceptación: 28 de febrero de 2021 y 20 de octubre de 2021

DOI: https://doi.org/10.46583/scio_2021.21.825

Resumen: A raíz de los avances realizados en neurociencia y sus implicancias en el derecho en general, y el derecho penal en particular, nos proponemos a evaluar si la normativa de los tratados internacionales sobre derechos humanos es suficiente para cubrir los principios que surgen de sus postulados, o si es necesaria una ampliación (ya sea interpretativa o normativa) en Latinoamérica. Realizaremos un breve repaso de los avances en el campo del neuroderecho y consideraremos algunas de las propuestas más destacadas. Trataremos el derecho a la

${ }^{a}$ Facultad de Ciencias Jurídicas y Sociales. Universidad Nacional de La Matanza. Argentina.

${ }^{\mathrm{b}}$ Facultad de Ciencias Jurídicas y Sociales. Universidad Nacional de La Matanza. Argentina.

${ }^{*}$ Correspondencia: Universidad Nacional de La Matanza. Facultad de Ciencias Jurídicas y Sociales. Florencio Varela, 1903. San Justo 1754. Buenos Aires. Argentina.

E-mail : jmarinaro@unlam.edu.ar

${ }^{1}$ La presente publicación se realiza en el marco de los Proyectos de Investigación Programa I+D+i orientada a los retos de la sociedad "Derecho penal y comportamiento humano" (RTI2018-097838-BI00) concedido por el Ministerio de Ciencia, Innovación y Universidades de España, bajo la dirección del Prof. Dr. Eduardo Demetrio Crespo. https://blog.uclm.es/proyectodpch/; y el Proyecto de Investigación D-066 "Espacios de progresión de las Neurociencias en el Derecho: aplicación al campo de los Derechos Humanos, Derecho Penal, Ejecución de la Pena, Neurociencia Forense y Neurotecnologías" del Departamento de Derecho y Ciencia Política de la Universidad Nacional de La Matanza, San Justo, Buenos Aires, Argentina, dirigido por el Prof. Dr. José Angel Marinaro. 
libertad cognitiva, la privacidad y la integridad mentales y la continuidad psicológica. Finalmente, proponemos una ampliación del bloque convencional interamericano de derechos humanos para dar respuesta a los nuevos posibles conflictos de derechos y garantías.

Palabras clave: neurociencias, neuroderecho, neuroética, derechos humanos, neurotecnologías.

Abstract: As a result of the advances made in neuroscience and its implications in law in general, and criminal law in particular, we propose to evaluate whether the regulations of international human rights treaties are sufficient to cover the principles that arise from their postulates, or if an extension is necessary (either interpretative or normative) in Latin America. We will carry out a brief review of the advances in the neuro-law field, and we will consider some of the most outstanding proposals. We will address the right to cognitive freedom, mental privacy, mental integrity and psychological continuity. Finally, we propose an expansion of the inter-American human rights conventional bloc to respond to new possible conflicts of rights and guarantees.

Keywords: neurosciences, neurolaw, neuroethics, human rights, neurotechnologies.

\section{$\S 1$ INTRODUCCIÓN}

La mayoría de las novedades neurocientíficas viene impulsada por el desarrollo vertiginoso de aquellas técnicas que tienen por objetivo común visualizar la dinámica del funcionamiento cerebral, detectando las funciones principales o críticas que poseen determinadas áreas.

A modo de ejemplo, es dable mencionar las numerosas publicaciones que se han realizado a tenor de la corteza prefrontal, en las que se indican que tienen un rol crítico en el desempeño de las denominadas funciones ejecutivas: en el control de los impulsos (Pliszka et al., 2006; Schulz et al., 2004), en la regulación de las emociones (Hoaken et al., 2007), en la ponderación de los riesgos y en el razonamiento moral (Giedd, 2008: 340; Harenski y Hamann, 2006; Mendez, 2006; Moll et al., 2005; Moll y De Oliveira-Souza, 2007), lo que ha tenido especial incidencia en el derecho penal juvenil por la inmadurez 
de dicha área (Burnett et al., 2010; Cohen et al., 2016; Mercurio, 2012: 54; 2014: 120; Mercurio et al., 2019; entre muchos otros).

Desde el neuroderecho, todo cuanto sea develado del pensar, sentir, juzgar y decidir de los seres humanos tiene que enmarcarse en el contexto de los sistemas normativos, precedidos de un análisis ético sobre el modo de obtención de los datos y sus usos.

En este trabajo nos proponemos, dentro del área latinoamericana, iniciar un estudio que nos permita discernir si los tratados internacionales que han positivizado los derechos humanos otorgan cobertura suficiente ante estos nuevos desafíos.

Al mismo tiempo, analizaremos si las propuestas más relevantes sobre este punto que promueven reglamentar nuevos derechos resultan necesarias o adecuadas.

\section{§2. NeURoteCnOlogías: SOMERA ENUMERACiÓN Y ESTAdO DEL ARTE}

La observación de la actividad cerebral ha sido posible en estos últimos tiempos gracias al considerable desarrollo de diversas técnicas. Siguiendo los lineamientos dados por Bunge (1999: 161) y Díaz (2008), sin pretender agotar la temática, las clasificaremos como de "espacio" y de "tiempo".

En el primer caso, las más conocidas son las denominadas IRMf (imagen por resonancia magnética funcional, o fMRI por sus siglas en inglés) y TEP (tomografía por emisión de positrones), entre otras. En el segundo caso, podemos mencionar al EEG (electroencefalograma) y el MEG (magneto encefalograma). Nos permitimos sugerir al lector una lista profunda y detallada en Asbury y Detre (2019) en tanto que, sobre tecnologías vinculadas a inteligencia artificial, Miró-Llinares (2018) brinda un panorama erudito y muy completo.

Dentro de las espaciales, la IRMf es la más famosa de todas. Dicha técnica se elabora a partir de la medición de las diferencias entre las propiedades magnéticas en sangre cuando esta fluye por las distintas regiones cerebrales (Gibson et al., 2007), marcándose la hemoglobina con un isótopo radiactivo que hace visible la zona mayormente irrigada. 
Por lo tanto, estas pruebas no miden la actividad neuronal directamente, sino que más bien se utiliza el flujo sanguíneo como una medida indirecta de dicha actividad. Esto último puede generar problemas epistémicos si las imágenes del IRMf son analogizadas erróneamente por las fotografías (Roskies, 2008). Sin perjuicio de ello, aún con sus limitaciones (Martinez et al., 2013) y posibles falsos positivos (Eklund et al., 2016), la IRMf es la última y más sofisticada técnica para examinar el funcionamiento cerebral.

Las denominadas temporales no son tan estimadas, y si bien no podemos ser absolutos en este punto, podría acusarse a la falta de un software lo suficientemente adecuado para el procesamiento de datos (cuya demora o delay provocaría un elevado de margen de falsos positivos). Sobre esta nomenclatura, la expresión inglesa familywise error (o FWE por sus siglas) expresa mejor la idea.

Logothetis (2008: 876-877) formula un elogio en torno a las bondades de la IRMf en neurociencias cognitivas, aunque con cierto tono de precaución orientado a la interpretación de los datos que se obtienen, dado que sus conclusiones a menudo ignoran las limitaciones reales de la metodología.

Dentro del estado del arte de las principales hipótesis neurocientíficas con impacto en el derecho, y aun realizando un análisis somero, no puede dejar de mencionarse el denominado problema "mente-cerebro" (Atmanspacher, 2014; Bunge, 1999; Giménez Amaya y Murillo, 2007; Lezaún, 1988; Lukomski, 2007; Martínez Freire, 1999; Pardo y Patterson, 2011; Sanguineti, 2005) y los cuestionamientos al libre albedrío (Muñoz Ortega, 2015).

El primero redunda en afirmar si el cerebro y la mente son entidades diferentes (por lo general, el primero está asociado a lo físico y el segundo, a lo metafísico) o si, por el contrario, ambos son una misma entidad. Los que afirman que se trata de entes diferentes suelen indicar que no es neurocientíficamente posible comprender la conciencia humana limitándose a lo físico o lo biológico (Pardo y Patterson, 2011: 7-8). Por el contrario, los que sostienen la unicidad suelen expresar que no se encuentra probada científicamente la existencia de entidades metafísicas ni de conciencia humana en ausencia del cerebro. Más aún, estudios realizados sobre pacientes con diversas patologías cerebrales (ya sea endógenas como exógenas) demuestran que cualquier daño en el cerebro afecta notoriamente a la funcionalidad del cuerpo, las funciones 
ejecutivas, entre otras, y en consecuencia a la experiencia de conciencia (como puede observarse en el caso reseñado en Burns y Swerdlow, 2003).

En este punto podemos ver con claridad cómo muchos de los debates que se realizan en torno a las neurociencias no son más que viejas discusiones reavivadas por los progresos en esta área, y que algunas veces no aportan novedad. Es que afirmar que no es posible comprender la conciencia humana a través de lo físico o biológico implica aseverar la existencia de objetos, seres o fuerzas inmateriales. Y si bien queda mucho terreno por recorrer para comprender el desarrollo y funcionamiento del cerebro humano, no es menos cierto que la discusión científica sobre la existencia de Dios no pertenece a este siglo.

En lo que respecta al libre albedrío, estudios llevados a cabo por Libet (1999) sugerirían que la "sensación de libre albedrío" sería en realidad una "ilusión", dado que la toma de decisiones a nivel cerebral es realizada de manera "no consciente". Como la temática no es el foco del presente artículo, nos limitaremos a indicar que dichos estudios han sido severamente cuestionados (tanto por cuestiones técnicas como metodológicas), pues existen importantes investigaciones que desacreditan las conclusiones a las que llegó (para más detalle sobre la cuestión, recomendamos Muñoz Ortega, 2016).

\section{§3. Neurocrimen: ¿SE PUEDE PIRATEAR EL CEREBRo?}

Debemos empezar aquí definiendo lo que se conoce como interfaz cerebrocomputadora (BCI, por sus siglas en inglés). En Gutiérrez Martínez et al. (2013: 63) se indican que su idea principal “... es capturar las manifestaciones eléctricas, magnéticas o de otro tipo de la actividad cerebral de los deseos de comunicación del usuario y traducirlas en órdenes que son interpretadas y ejecutadas por una computadora u otro dispositivo. (...) se consideran una herramienta con un enorme potencial para (...) rehabilitación a pacientes con discapacidad neuromotora". También que "Las BCI establecen una conexión directa entre las áreas del cerebro que siguen siendo dispositivos funcionales y de asistencia, como prótesis y ortesis eléctricas para brazos y piernas, sillas de ruedas motorizadas, órganos sensoriales artificiales y otras tecnologías para la restauración de las funciones motoras y sensoriales" (Levitskaya y Lebedev, 
2016; traducción propia). Se los clasifica en no invasivos (basados en EEG de superficie) e invasivos (si requieren intervención quirúrgica).

Se observan las aplicaciones de esta tecnología en diversas áreas, como el deletreador (que permite la selección de letras y símbolos), el control remoto de dispositivos (como una televisión, video o aire acondicionado) y el control de la silla de ruedas o de prótesis. También se menciona la posibilidad de rehabilitación de determinadas patologías neurológicas (como la hiperactividad por falta de atención, los problemas de memoria o la epilepsia) y, como salida comercial, se menciona un sistema capaz de procesar la escritura (Gutiérrez Martínez et al., 2013: 66-67). Cabe recalcar que todo ello sería posible por medio de electroencefalogramas y sin otra actividad del usuario más allá de pensar.

La estimulación magnética transcraneal (TMS, por sus siglas en inglés), que resulta ser una forma no invasiva de estimulación de pequeñas regiones cerebrales, ha logrado convertirse en un dispositivo portátil efectivo en el tratamiento de la migraña (Lefaucheur et al., 2014).

En lo que respecta a tratamientos invasivos, la estimulación cerebral profunda (DBS, por sus siglas en inglés) consiste en la implantación neuroquirúrgica de un dispositivo médico que envía impulsos eléctricos a áreas subcorticales dirigidas con el objetivo de generar actividad. Rasche, Rinaldi, Young y Tronnier (2006) demostraron que esta es útil para el tratamiento de algunas enfermedades (dolor crónico y dolor neuropático). Más recientemente, Tronnier y Rasche (2014) afirmaron que se trata de una terapia cada vez más utilizada para varias afecciones neurológicas, entre ellas la enfermedad de Parkinson, la distonía y el temblor esencial (especialmente cuando el paciente no ha tenido una respuesta positiva a otras terapias menos invasivas).

A su vez, debe indicarse que en la actualidad existen investigaciones que se encuentran en curso con el objetivo no solo de aplicar este tipo de tecnología para la rehabilitación de pacientes, sino también con otros fines, como pueden ser los videojuegos simulados mediante realidad virtual. En este sentido, en Yuan et al. (2010) predicen que estos neurodispositivos reemplazarán gradualmente a los teclados, las pantallas táctiles, los ratones, y los comandos de voz como la manera preferida de los humanos de interactuar con las computadoras. 
Más aún, existen los cognitive enhancement o mejoras cerebrales (nootrópicos si se tratan de medicamentos) que son mejoras dirigidas a aumentar el rendimiento cognitivo de seres humanos sanos (Normann y Berger, 2008).

En la publicación realizada por Ienca y Haselager (2016) se propone el término brain-hacking para referirse a la posibilidad de acceder de manera no autorizada a una interfaz cerebro-computadora (u otro dispositivo de ingeniería neuronal) con el propósito de obtener y/o manipular información proveniente del cerebro de un usuario. Si bien no hay una traducción que englobe todo el significado de la palabra, podríamos hablar de "pirateo cerebral". Así, "hackear" hace referencia a la capacidad de introducirse en un sistema informático ajeno (para manipularlo, obtener información, etc.) con fines lícitos, y que "crackear" hace referencia a esa misma capacidad cuando es utilizada con fines delictivos.

Estos autores también expresan que la velocidad a la que se producen los avances tecnológicos (que, a su vez, no son acompañados de regulaciones legales) convierten a los objetivos de la seguridad informática (principalmente la confidencialidad, la integridad y la disponibilidad de datos) en difíciles de alcanzar. Y, dentro de la información que se hace accesible (y, por lo tanto, expuesta al cibercrimen), la biológica es crítica, y lo es aún más si hablamos del cerebro. Potencialmente, "el uso indebido de dispositivos neurales con fines cibernéticos puede no solo amenazar la seguridad física de los usuarios, sino también influir en su comportamiento y alterar su autoidentificación como personas" (Ienca y Haselager, 2016: 119; traducción propia).

Para aclarar las cosas, indicamos que actualmente no es posible tener un acceso directo al cerebro o su información. Más bien, conforme fuera explicado anteriormente, el acceso a este sí podría darse de forma indirecta a través de alguno de los dispositivos controlados neuronalmente (BCI o tecnologías similares) que, valga la redundancia, existen y se utilizan hoy en día.

Ienca y Haselager (2016: 120) mencionan a empresas pioneras como Emotiv y Neurosky, que pretenden comercializar interfaces cerebro-máquina no invasivos y fáciles de usar para videojuegos, televisiones interactivas, sistemas de control de manos libres (en igual sentido, Bonaci, Calo y Chizeck, 2014); el accesorio Xwave de iPhone, que permite la lectura de ondas cerebrales; 
compañías como Nielsen, que están utilizando aplicaciones de BCI para evaluar mejor las necesidades y preferencias del cliente; o el propio Gobierno de Estados Unidos, que se encuentra desarrollando estas tecnologías con usos militares, entre otras. También mencionan varios experimentos en los que se ha conseguido hackear dispositivos BCI, con escalofriantes resultados, y hacen un análisis del ciclo de las BCI indicando los posibles ataques que podría recibir en cada fase.

Como puede observarse, cada vez con mayor certeza es posible captar las preferencias del público en general. Las grandes empresas pueden establecer diferencias funcionales en el cerebro de las personas que consumen sus productos y de la mano de esos resultados fueron diseñando sus estrategias de marketing. Nació así otra de las tantas derivaciones de la neurociencia: el neuromarketing. En el presente, tal como nos indican Ienca y Andorno (2017), varias empresas multinacionales, como Google, Disney, CBS y Frito-Lay, utilizan servicios de investigación de neuromarketing para medir las preferencias e impresiones de los consumidores en sus anuncios o productos. Esta posibilidad de "minar la mente", indican, puede usarse potencialmente no solo para inferir preferencias mentales, sino también para originar, imprimir o desencadenar esas preferencias.

Paralelamente, y tal como se indicó en la introducción, debe recordarse que las técnicas de neuroimagen para mapear el funcionamiento del cerebro también se han probado capaces para obtener información sobre las intenciones, puntos de vista y actitudes de las personas. Ienca y Haselager (2016: 128) indican que un ataque cibernético a un dispositivo neuronal podría no solo modificar el rendimiento del dicho positivo, sino potencialmente modificar la propia toma de decisiones (si se trata de DBS, TMS o similar).

Es harto sabido que las modificaciones legales ocurren con posterioridad a los nuevos acontecimientos, aunque al mismo tiempo debe apuntarse que la creación de nueva legislación con fines de regular situaciones aún inexistentes posee un altísimo grado de dificultad que lo convierte en una tarea titánica o, quizás, lisa y llanamente imposible. Pero, si bien predecir el futuro escapa al derecho, entendemos que una saludable postura epistémica y prudente debe conducirnos a estar atentos a los avances científicos y tecnológicos con sus posibles aplicaciones prácticas. 


\section{§4. ÉTICA Y DERECHOS HUMANOS EN JUEGO}

Dicho lo anterior, es necesario abordar el asunto desde una perspectiva ética. La pregunta que debemos responder, entonces, versa sobre la ética en la utilización de estas nuevas tecnologías.

Como se viene analizando, es fácil detectar que su utilización puede servir tanto para causas nobles (como la rehabilitación de personas que padecen algún tipo de enfermedad), como también para crímenes de una gravedad tal que solamente cabría imaginarlos en la literatura de ciencia ficción. La mera idea de que pirateando algún dispositivo neuronal se pueda afectar a la toma de decisiones de una persona resulta difícil de dimensionar.

A este tipo de dilemas se los suele denominar "de uso dual" dado que, dependiendo de su uso, una misma tecnología puede ser utilizada para buenos o malos propósitos. Esto tiene poco de novedoso, ya que los avances científicos en varios ámbitos han presentado este dilema. Quizás el más conocido históricamente sea el desarrollo de la física nuclear que, si bien proveyó de varios descubrimientos (como la energía nuclear), en agosto del año 1945 permitió el bombardeo a Hiroshima y Nagasaki. La solución que se suele aplicar es reglamentar el uso de la tecnología para maximizar sus aspectos positivos y reducir o eliminar los negativos (en el ejemplo, el propósito que tuvo el Tratado de No Proliferación Nuclear).

Con esto, y dejando un análisis más acabado para futuros artículos, entendemos que no sería correcto prohibir esta tecnología por sus posibles implicancias negativas. La salida es una correcta reglamentación.

Ante este estado dinámico, hemos de interrogarnos si los preceptos relacionados con los derechos humanos se encuentran en condiciones de proveer adecuada protección contra los empleos inadecuados, que conduzcan a menoscabar variados aspectos relacionados con el ser humano individual y socialmente considerado.

Dicho esto, analizamos a continuación la propuesta de Ienca y Andorno (2017: 8). De manera preliminar, estos autores sugieren que las neurotecnologías probablemente sigan el camino trazado por el avance de otras disciplinas, haciendo hincapié en la tecnología genética. En este sentido, mencionan la Declaración Universal sobre el Genoma Humano y los Derechos Humanos, 
realizada por la Unesco en el año 1997, y la Declaración Internacional sobre los Datos Genéticos Humanos, realizada por esa misma organización en el año 2003.

Este paralelismo, más allá de su posible acierto, encierra un error. Generalmente, cuando hablamos de derechos humanos nos referimos al derecho positivo o, más específicamente, a tratados internacionales sobre derechos humanos que el país en cuestión haya firmado y ratificado o, de algún modo, adoptado como norma vigente en algún ordenamiento interno. Las declaraciones de la Unesco, en la medida en que permanezcan solamente como declaraciones, no poseen obligatoriedad en sí mismas, más allá de que un juez podría considerarlas como referencia para interpretar otras normas de derechos humanos que sí tengan vigencia.

Sin perjuicio de ello, también debe indicarse que los organismos estatales o profesionales internos de cada país suelen observar este tipo de declaraciones al momento de reglamentar prácticas o técnicas ligadas a la salud sin por ello adoptarlas.

Hecha esa aclaración, Ienca y Andorno (2017) afirman la insuficiencia de los derechos humanos existentes para responder a estos problemas emergentes y proponen cuatro nuevos derechos a estos fines: libertad cognitiva, privacidad mental, integridad mental y continuidad psicológica.

En primer lugar, definen como libertad cognitiva (o autodeterminación mental) el derecho de las personas a utilizar neurotecnologías emergentes y, a la vez, su protección contra el uso coercitivo y sin consentimiento de tales tecnologías. Mencionan que este nuevo derecho sería una suerte de actualización de la denominada libertad de pensamiento, dándole un carácter multidimensional. Afirman también que, siendo el sustrato neurocognitivo de todas las demás libertades, no puede ser reducido a los derechos existentes, lo que otorga una suerte de mayor jerarquía a nivel conceptual.

En segundo lugar, la privacidad mental implica la protección contra el acceso ilegítimo a la información de sus cerebros y prevenir la filtración indiscriminada de datos cerebrales a través de la infosfera. Explican que el actual derecho a la privacidad no cubre en su totalidad a la privacidad mental debido a que la definición de privacidad no presenta un consenso (puesto que se incluyen en él de manera dispar el derecho a controlar el acceso a la información 
personal, a nuestros cuerpos, o a lugares privados específicos). En ese sentido, aseveran que es muy probable que las neurociencias se conviertan en una de las nuevas áreas en las que el derecho a la privacidad deba desempeñar un papel fundamental e inesperado (a este respecto, véase Shen, 2013).

En tercer lugar, la integridad mental es entendida como el derecho de las personas con enfermedades mentales a acceder a esquemas de salud y recibir el tratamiento o apoyo psiquiátrico correspondiente $\mathrm{y}$, a la vez, en similitud con la integridad física, como el derecho a proteger su dimensión mental de cualquier daño potencial. Conectan este derecho con las explicaciones dadas anteriormente respecto del denominado neurocrimen y brain-hacking, aunque lo extienden hacia otro tipo de alteraciones no autorizadas del cerebro (como podrían ser su aplicación para mejorar el rendimiento de soldados con fines militares, o contra prisioneros de guerra para aplicarles lavado de cerebro u otras intervenciones invasivas).

Mencionan que este derecho tiene conexión con las muchas técnicas que han sido desarrolladas con la finalidad de borrar memorias del cerebro de una persona. Es interesante mencionar a este respecto el artículo realizado por Merkel (2013: 91) en el que se pregunta si una persona que debía testificar en un caso y borra sus recuerdos sobre este comete el delito de falso testimonio, y en el que la alteración de la memoria y sus registros se viene estudiando desde hace décadas (Loftus y Davies, 1984).

En cuarto lugar, indican que los cambios en la función cerebral causados por la estimulación cerebral también pueden causar alteraciones involuntarias en estados mentales críticos para la personalidad y, por lo tanto, pueden afectar a la identidad personal de un individuo. En ese sentido, la continuidad psicológica es definida como el derecho de la persona a mantener la percepción de su propia identidad.

En paralelo con lo que se indica sobre la eliminación de recuerdos, estas técnicas pueden afectar a la identidad de una persona al eliminarlos, alterarlos, agregarlos o reemplazarlos de manera selectiva, siendo estos relevantes para el autorreconocimiento de las personas. Aseveran, entonces, que los nuevos conocimientos y tecnologías en el campo de la neurociencia ofrecen claramente nuevas y más eficientes posibilidades para llevar a cabo cambios de personalidad sin consentimiento. 
Resumen que el derecho a la continuidad psicológica en última instancia tiende a preservar la identidad personal y la coherencia de la conducta del individuo frente a modificaciones no consensuadas por parte de terceros. Protege la continuidad en los pensamientos, preferencias y elecciones habituales de una persona al proteger el funcionamiento neuronal subyacente.

Si bien algunos pueden considerarlo como parte del derecho a la identidad, estos autores indican que este derecho excede esta, dado que incluye etapas previas a su formación.

En diferentes momentos, al tratar estos derechos, los autores se preguntan si estos son absolutos o relativos y, consecuentemente, si sería aceptable ingresar compulsivamente en la mente de una persona para extraer información sobre un crimen que ocurrió u ocurrirá, o modificar su psiquis de manera forzosa para introducirle un razonamiento moral a través de mejoras cerebrales (siempre que estas sean seguras y eficaces). Indican que reconocer estos derechos como humanos no significa necesariamente que tengan carácter absoluto, y ejemplifican con casos en los que su utilización sería justificada.

Imaginemos todas las consecuencias que la temática podría acarrear si las mejoras cerebrales, en consonancia con propósitos de prevención especial, se piensan como formas coactivas de abordaje.

Con una mirada crítica del artículo recién analizado, Sommaggio y Mazzocca (2020) proponen algunos nuevos puntos de vista que son importantes de mencionar. Tras realizar un estudio sobre el impacto de las neurotecnologías (imágenes, estimulaciones y mejoras cerebrales), analizan la posición de Ienca y Andorno y establecen que uno de los primeros problemas en abordar es la definición de lo que se entiende por libertad cognitiva.

Citando a Bublitz (2013), explican que la principal diferencia entre esta última y la ya conocida libertad de pensamiento sería que la libertad cognitiva incluye el derecho a alterar el estado mental propio con la ayuda de dispositivos neurotecnológicos, como así también negarse a su uso. También lo definen como el derecho de cada individuo a pensar de forma autónoma e independiente, a utilizar el conjunto completo de sus capacidades mentales y a participar en diversas formas de pensamiento. Así, dado que aseguraría la posibilidad de controlar nuestra propia conciencia, debería permitir evitar un ataque de una corporación económica o de una legislación política coercitiva. 
Dicho esto, consideran que puede establecerse una definición por medio de una formulación negativa o positiva. La negativa tiene por objetivo proteger a los individuos de abusos de terceros en la utilización de esta tecnología. Ejemplifican esto con la posibilidad de que una entidad policial, médica o comercial intente realizar un uso forzoso de las neurotecnologías en prisioneros, pacientes o consumidores (respectivamente).

Exponen que ha habido voces (como en Greely, 2012) que se han expresado a favor del uso compulsivo de tratamientos para eliminar o modificar comportamientos antisociales o enfermedades mentales tanto en prisioneros como en determinados pacientes psiquiátricos. Esto se debe a que, en su opinión, en un futuro próximo la neurociencia proporcionará la capacidad de modificar comportamientos no deseados, cambiando la base neurológica de los individuos.

Siguiendo con el razonamiento de los autores, la formulación positiva sostiene que las neurotecnologías existentes deberían estar ampliamente disponibles para cualquiera que las desee. Su objetivo, de hecho, no es tanto defender la mente humana de interferencias no deseadas, como garantizar a cualquier persona la posibilidad de hacer lo que desee de su propio potencial cognitivo. Explican que esta formulación provoca un posicionamiento respecto del debate entre transhumanismo y bioconservación de la especie humana, dado que su uso podría tener consecuencias profundas e impredecibles en la sociedad. También mencionan que su uso podría profundizar las disparidades económicas y sociales entre los más ricos (quienes podrán acceder a estas tecnologías) y los más pobres (quienes no podrán), e incluso provocar que en determinadas actividades o profesiones su uso se vuelva necesario u obligatorio.

Dicho esto, se preguntan si el estado debería intervenir para prevenir su uso y afirman que la definición por medio de una formulación positiva permitiría la utilización de las mejoras cerebrales.

Seguidamente, los autores realizan un análisis en relación con los derechos humanos en juego. Al igual que Ienca y Andorno, afirman la misma definición de derecho humano y mencionan la "inflación de derechos". También analizan los cuatro derechos que ellos proponen, y la descripción que hacen de cada uno de ellos.

En sus conclusiones, afirman la necesidad de definir conceptualmente qué significa libertad cognitiva, y las consecuencias que implicaría una formulación 
positiva o negativa. Compartimos con estos autores la importancia y urgencia que le han dado a esta cuestión.

Para finalizar este apartado, queremos resaltar la tarea de Rafael Yuste y su equipo Brain Initiative que, aunque orientados primariamente a aspectos neurobiológicos, también hicieron trabajos muy relevantes sobre la ética de las neurotecnologías, destacando lo relativo a la privacidad y el consentimiento, voluntad e identidad, mejoras cerebrales, y los sesgos (Yuste et al., 2017; Yuste y Goering, 2016). Expresaron que las nuevas neurotecnologías pueden alterar la identidad humana y la sociedad de manera profunda, abogando por la integración temprana de la ética en la neurotecnología. También es destacable el proyecto europeo conocido como Human Brain Proyect que en su estructuración originaria ha previsto la conformación de un Comité de Ética de la Investigación y un Comité de Aspectos Éticos, Legales y Sociales, que asesoran en esas áreas al proyecto general.

\section{§5. NeCESIDAD DE CREAR NUEVOS DERECHOS}

Ienca y Andorno (2017) eligieron adoptar una concepción práctica amplia de los derechos humanos como la que sostiene Beitz (2011). Indican que no cualquier derecho debe ser considerado como un derecho humano, dado que ello provocaría la llamada "inflación de derechos", definida como la tendencia objetable a etiquetar todo lo que es moralmente deseable como un derecho humano.

Para evitar esto, siguiendo lo expuesto por Nickel (2014), afirman que es necesario aplicar un examen para verificar si el "nuevo derecho" se encuentra justificado. Para ello, no es necesario solamente lidiar con un "bien" muy importante, sino también responder a una amenaza común y grave para ese bien, imponiendo cargas a los destinatarios que son justificables y no mayores de lo necesario, y que sea factible en la mayoría de los países del mundo.

Desde una perspectiva latinoamericana, la supuesta negatividad de la "inflación de derechos" es debatible. Argentina ha adoptado diversos tratados internacionales de derechos humanos, a doce de ellos le ha dado jerarquía constitucional (art. 75 inc. 22 de la Constitución Nacional de Argentina), y 
a estos se le suman los derechos humanos que ya se encontraban en la carta magna. Este ejemplo se repite de manera similar en otros países de la región.

En otras palabras, actualmente a nivel latinoamericano ha habido una gran cantidad de reconocimientos de derechos humanos, y esta situación no ha producido efecto negativo alguno. Tal es así que la denominada "inflación de derechos" no es un tema de debate actual en la región. Por el contrario, los debates suelen darse en relación con el reconocimiento de más derechos humanos.

Luego, el debate sobre qué entendemos por derechos humanos, al menos en Latinoamérica, es bastante más complejo que el propuesto por los autores citados. Históricamente, en nuestra región, ha sido objeto de arduo debate la pugna entre las posturas iusnaturalista e iuspositivista con relación a estos derechos, con una inmensidad de autores y de opiniones encontradas. En tanto en las praxis, los recurrentes Gobiernos de facto han provocado graves afectaciones de esos derechos fundamentales. En este sentido, resulta ilustrativo el trabajo de Zaffaroni (2012).

Como consecuencia, muchos autores sostienen que, como los derechos humanos se han positivizado, la vieja discusión hoy carece de sentido continuarla (en ese sentido, Bobbio, 2009; Ferrajoli, 2016). La mera idea de que los avances de la tecnología provoquen nuevas situaciones en las que los derechos humanos actuales no son capaces de dar respuesta rompe con esa estructura y revive viejas discusiones.

La diferenciación entre libertad cognitiva y libertad de pensamiento, sin embargo, no pareciera estar justificada. No se observa, por lo menos jurídicamente, un motivo por el cual deberían separarse, cuando ambas se refieren exactamente a lo mismo: el derecho a que cada ser humano piense lo que desee pensar y que otros no puedan intervenir en sus pensamientos. Sin duda, dicho derecho debe ser actualizado para incluir nuevas formas posibles de vulneraciones, pero eso no implica necesariamente que estemos en presencia de nuevos derechos. Por citar un ejemplo: el mero hecho de que ya no usemos monedas de oro sino tarjetas de crédito no ha cambiado el nombre al derecho a la propiedad, sino que ha sido actualizado conforme a las nuevas formas de transacción. En ese sentido, quizás sería interesante hablar de libertad cognitiva solo para enfatizar la imperiosa necesidad de actualización. 
Entendemos que las propuestas de Ienca y Andorno, Yuste, y Sommaggio y Mazzocca se hallan justificadas en la idea de incorporar las categorías que diseñan en su trabajo. Aun cuando el concepto de libertad posee límites difusos, cierto es que los modos de afectación explicados anteriormente son novedosos en cuanto a los medios en que dichas improntas pueden producirse.

Es necesario, sin embargo, realizar algunas salvedades. La idea de privacidad mental posee algunos detalles conceptuales que necesitan ser analizados y profundizar en ellos. Obsérvese que, hasta la fecha, cuando hablamos de privacidad (en mayor o menor medida) nos estamos refiriendo a objetos (físicos o virtuales) que no deberían poder ser alcanzados por terceros. Este concepto, que hace algunas pocas décadas se plasmaba principalmente en la protección de cartas, diarios personales o papeles privados, tuvo que ser actualizado y hoy en día abarca datos e información contenida en las redes (Ferrajoli, 2016). Pero este cambio, más que una modificación del derecho existente fue una adaptación por analogía: en el derecho se le dio similar tratamiento al correo y al correo electrónico, a los papeles privados y a los archivos de texto que almacenamos en ordenadores, etc.

La posibilidad de acceder a pensamientos, entonces, se aprecia como una situación de otra magnitud. Equipararlo a la idea (por cierto, difusa) de la privacidad sería desvalorizar la proporción de los derechos en juego. La libertad de pensamiento o libertad cognitiva debe ascender a lo más alto de la escala de derechos humanos, equiparándose al derecho a la salud y solo pudiendo ser superado por el derecho a la vida. Y si bien la temática relativa a qué derecho humano es prioritario sobre otro (y las consecuencias jurídicas que ello provoca) exceden los límites de este artículo, debemos dejar asentada su gran relevancia.

Por otro lado, la idea de continuidad psicológica posee problemáticas insalvables en lo concreto. Definir, establecer o determinar por un tercero (y con algún grado de certeza) cuál es la identidad personal de cada individuo, o cuál es su percepción de ella, es simplemente imposible. Solamente nos podemos conformar con un relato incontrastable que expresa cada ser humano.

Además, la idea de "continuidad" implica cierto grado de permanencia o estabilidad, es decir, lo opuesto a la dinámica de la plasticidad neural. Sin embargo, los seres humanos somos cambio constante en un devenir que ine- 
vitablemente concluye con la muerte. Lo permanente en el ser humano no es más que una ilusión, una apariencia, un relato, que científicamente es falso.

Entonces, si lo permanente no existe, ¿cómo podríamos afirmar la existencia de un derecho a permanecer psicológicamente iguales?

También debe decirse que la afectación voluntaria o involuntaria de las funciones cerebrales posee consecuencias que son altamente relevantes para el ámbito jurídico. No estamos en presencia de nuevas situaciones que hayan sido previsibles años atrás cuando las normas de derechos humanos fueron transformadas en derecho positivo; tampoco estamos ante un mero cambio de aspectos secundarios, y mucho menos situaciones hipotéticas pertenecientes al mundo de la ciencia ficción.

Así como la modificación genética parecía imposible y en la actualidad nos encontramos consumiendo diariamente productos transgénicos, las modificaciones al cerebro humano son un hecho y están aumentando exponencialmente.

\section{§6. EJECUCIÓN DE LA PENA Y LOS TRATAMIENTOS FORZOSOS}

Siguiendo con la idea antes esbozada, Ienca y Andorno (2017) mencionan de manera muy somera la realización de algún tipo de tratamiento médico compulsivo de índole penal. Dan varios ejemplos, como sería ingresar en la mente de una persona para extraer información sobre un crimen que ocurrió u ocurrirá, o modificar su psiquis para introducirle un razonamiento moral a través de mejoras cerebrales (siempre que estas sean seguras y eficaces).

En este aspecto, el análisis sobre la cobertura que brindan los derechos humanos en relación con las nuevas tecnologías tiene un apartado separado cuando hablamos de la privación de la libertad como condena por la comisión de un crimen.

La enumeración de las diversas técnicas que hicimos al inicio debe traerse a este terreno, donde los sujetos privados de libertad pueden ser objeto de sus aplicaciones, atendiendo tanto a las necesidades terapéuticas como rehabilitadoras, libertarias o lúdicas. 
Los mencionados son los espacios de análisis: las terapias para el abordaje de patologías, las aplicaciones en casos de sujetos que consientan los empleos en sus humanidades de cara a su acceso a estamentos de rehabilitación y de allí en más, al logro de reglamentarias condiciones preegreso. El reciente estudio de Ligthart (2019) sobre métodos coercitivos, analizados a la luz de la privacidad, sugiere que puede defenderse la necesidad de un nuevo derecho humano europeo específico a la privacidad mental. A la vez, postula que el empleo de algunos tipos de neuroimagen coercitiva, bajo ciertas condiciones y garantías específicas, no contraviene el derecho a la privacidad del art. 8 del Convenio Europeo de Derechos Humanos.

Es en este punto donde se tiende a formar juicios futuros, esto es, estimaciones acerca de las probabilidades de reincidencia con base no ya en datos clínicos o el hallazgo de ciertos biomarcadores neurocognitivos (Aharoni et al., 2013), sino en la aplicación de neurotecnologías que en sus variadas especies puedan arrojar certeza en esas labores de predicción. Abundante literatura por estos días aborda los aportes procedentes de la Inteligencia Artificial. El empleo de algoritmos está siendo estudiado y, en el caso de la justicia norteamericana, ya están siendo empleados para las estimaciones o prognosis de reiteración delictiva con no pocas resistencias, ante la afectación de garantías propias del proceso criminal.

En similar sentido, Martínez Garay (2020) formula el siguiente interrogante: ¿es compatible con el derecho al debido proceso agravar la pena sobre la base de la valoración de riesgo hecha por un algoritmo, cuyo funcionamiento no se desvela al acusado porque está protegido como secreto de empresa?

Ienca y Andorno (2017) toman nota de lo hecho por Aharoni et al. (2013) y explican que en este último estudio se tomó un grupo formado por 96 prisioneros que fueron examinados por medio de IRMf mientras desempeñaban tareas en una computadora en las que tenían que tomar decisiones rápidas e inhibir reacciones impulsivas. Luego de cuatro años siguiéndolos, los resultados indicarían que aquellos que muestran una actividad baja en una región del cerebro asociada con la toma de decisiones y la acción tienen más del doble de probabilidades de cometer delitos nuevamente. Sobre este tema, también es interesante lo expuesto por Poldrack et al., (2018), para quienes la neuro- 
predicción ofrece un potencial para determinar algunos mecanismos causales, aun cuando estemos, por estos tiempos, lejos del ideal predictivo.

En otras palabras, estos autores afirman que las exploraciones cerebrales teóricamente pueden ayudar a determinar si ciertas personas condenadas tienen un mayor riesgo de reincidencia si son liberadas.

Una conclusión de este estilo es un peligro para la credibilidad neurocientífica, dado que realiza un salto al vacío desde un punto de vista metodológico. Podríamos resumir que muchas de las críticas a los experimentos "tipo Libet" (Muñoz Ortega, 2013) son analógicamente aplicables a este tipo de estudios. Sin embargo, debemos poner de manifiesto que la reincidencia es un problema multifacético y que intentar exponerlo como un único factor (baja actividad de una región cerebral) es un palmario y disfuncional reduccionismo.

Además, deja muchas preguntas relevantes sin respuesta adecuada. Cabría preguntarse si la baja actividad de esa región cerebral es causa o consecuencia de la privación de la libertad; si se analizaron o evaluaron otros factores que pudieran haber tenido una incidencia más importante; o si es posible realizar tratamientos o seguimientos luego de la liberación que reduzcan su probabilidad.

Todo lo relativo a la ejecución penal es uno de los segmentos más delicados de las prácticas apegadas al neuroderecho, no solo en las novedosas y dignas posibilidades de rehabilitar (resocializar, renormativizar, etc., de conformidad con el artículo 5.6 de la Convención Americana de Derechos Humanos), sino en las de determinar al tiempo de los eventuales egresos que prevén los regímenes legales, como también las estimaciones o proyecciones de reiteración. Es necesario debatir en qué casos sería válido que las modificaciones en la personalidad producto de intervenciones neurocientíficas pudieran incidir en una liberación anticipada (tanto para favorecerla como para impedirla), o incluso en la determinación de responsabilidad penal.

Por otro lado, nos encontramos por estos tiempos verificando que en nuestra región los informes sobre los que se sustentan las tramitaciones de libertades anticipadas son de tenor clínico exclusivamente y están basados en entrevistas, exámenes anacrónicos y en la subjetividad de los expertos. 
Del mismo modo en que puede acontecer en el medio libre, la aplicación de los tratamientos que empleen medios tecnológicos o de cualquier otra índole han de requerir el consenso del destinatario, al menos en los casos en que no medien impedimentos por razones patológicas.

Pérez Manzano (2011: 25) sostiene que la imposición del tratamiento o de medidas terapéuticas es contraria a la autonomía personal, a la identidad personal, al libre desarrollo de la personalidad, al derecho a la igualdad de trato y a la propia dignidad humana, de modo que los contenidos positivos de la prevención especial -resocialización- solo pueden imponerse de manera coactiva a inimputables, a quienes por definición no se les impone la pena y no se les reconoce responsables de sus actos.

En ese sentido, entendemos que tanto la libertad cognitiva como el derecho a la privacidad mental, a la integridad mental y a la continuidad psicológica no se suspenden por el hecho de hallarse cumpliendo condenas a penas privativas de libertad.

Pérez Manzano (2011: 25) lo explica claramente:

Una de las críticas fundamentales al contenido de la resocialización es precisamente que en el Estado de Derecho el respeto a la libertad ideológica, a la autonomía y al libre desarrollo de la personalidad, impiden que se imponga la asunción de valores, de modo que si sólo se puede exigir el respeto externo de las normas, sólo se puede ayudar a generar mecanismos y habilidades para conseguir dicho respeto externo con independencia de la no asunción de valores. Las medidas terapéuticas deben ir orientadas a ganar la autonomía que hace al individuo capaz de respetar las normas.

Compartimos la idea de esta autora en cuanto a que el cerebro no es un órgano más de nuestro cuerpo, es ante todo la base de nuestra conciencia, de nuestra idea del yo, estudiado en profundidad por Dehaene (2015). Allí se constituye nuestra subjetividad y presupuesto fisiológico de nuestra identidad por lo que cualquier consideración sobre las posibilidades de tratamiento neurológico debe tener en cuenta que es objeto de protección tanto constitucional como penal, y que por ello es un ámbito inmune a injerencias ajenas que el Estado debe ante todo no lesionar sino garantizar. 
Resulta particularmente desconcertante que las investigaciones sobre neurociencia aplicada a la ejecución penal tengan como objetivo tratamientos forzosos. Las herramientas neurocientíficas podrían ayudar y brindar nueva y mejor información sobre el tratamiento carcelario más adecuado para una determinada persona. Bajo la premisa del derecho como ciencia necesariamente interdisciplinaria, las nuevas técnicas neurocientíficas, junto con otras técnicas provenientes de otras áreas del conocimiento, podrían brindar una respuesta más acabada sobre cómo tratar a una persona que ha sido condenada por la comisión de un crimen.

En nuestro país, uno de los pioneros en estas temáticas ha sido Gustavo A. Arocena (2015a y 2015b). El autor reflexiona acerca de las contribuciones de las neurociencias, en general, y de la neurobiología, en particular, al derecho penal y a la configuración del tratamiento penitenciario orientado a la eficaz reinserción social del recluso. Analiza específicamente las alternativas de tratamiento médico y psicológico del comportamiento agresivo del delincuente.

Por lo demás, es necesario enfatizar que considerar el delito y la violencia (física o verbal) como inescindibles deja por fuera una enorme cantidad de delitos que no se cometen mediando la violencia, como son los denominados popularmente "delitos de guante blanco" (tráfico de influencias, fraude, lavado de dinero, cohecho, peculado, malversación de fondos, entre otros).

Teniendo especialmente en cuenta la legislación latinoamericana relativa a derechos humanos, como así también la historia de cada uno de los países de nuestros continentes, desde nuestra perspectiva la posibilidad de una intervención neurocientífica legalmente forzosa (ya sea a reclusos, pacientes, consumidores o humanos en general) se aprecia no solamente como inaceptable sino impensable. Tanto es así que todos los juristas latinoamericanos con los que hemos entablado conversaciones se han opuesto vehementemente a esta idea.

La perspectiva es simple: hemos vivido tantas dictaduras, tantas veces Gobiernos que empezaron siendo democráticos y terminaron no siéndolo; tantas veces hemos visto vulneraciones a derechos humanos, que no tenemos el mismo grado de confianza. Estos ejemplos, lamentablemente, han ocurrido bien hace cincuenta años bien hace unos pocos. Para nosotros, el Estado debe ser controlado, y se le ha de dar la menor cantidad de poder posible para cumplir sus fines. 
No concebimos la posibilidad de que se puedan realizar legalmente tratamientos médicos forzosos de esta índole porque todo lo que puede imaginarse que un gobierno dictatorial o pseudodemocrático haría con tal poder será hecho, si se le da la posibilidad. El riesgo es demasiado alto.

Dicho esto, entendemos también que el derecho debe tomar un papel protagónico en defensa de los seres humanos y sus cualidades intrínsecas. Las propuestas analizadas, más allá de las críticas que realizamos, cumplen con este rol y muestran un posible camino que seguir. Por ello, y en el sentido desarrollado, proponemos una ampliación del bloque convencional interamericano de derechos humanos (conformado tanto por los tratados internacionales como regionales) en la que se contemplen las perspectivas analizadas. Sería importante, además, que los organismos internacionales tomen nota de esta situación a fin de garantizar su uso no bélico y evitar la vulneración de derechos humanos, especialmente los vinculados con la niñez.

En nuestra región, los desarrollos de las neurociencias y del neuroderecho son básicamente especulativos con escasa producción de fundamentos empíricos propios (al respecto, véase García-López et al., 2019), ya sea por insuficiente financiación privada o por recortes presupuestarios de determinados Gobiernos latinoamericanos que parecen olvidar que, sin ciencia ni tecnología, se pierde soberanía. Una bienvenida excepción es el proyecto de reforma constitucional de Chile, que prevé la incorporación de la integridad mental como un derecho fundamental, generado con el firme y sostenido apoyo del Senado de ese país hermano.

Finalmente, el análisis que aquí formulamos forma parte de una labor en progreso. Se trata de dar pasos concretos en la estructuración de normas de derechos humanos que insertar en los moldes legales tanto internos como supranacionales.

Queda abierta la discusión.

\section{REFERENCIAS BIBLIOGRÁFICAS}

Aharoni, E., Vincent, G. M., Harenski, C. L., Calhoun, V. D., Sinnott-Armstrong, W., Gazzaniga, M. S., Kiehl, K. A. (2013). Neuroprediction of future 
rearrest. Proceedings of the National Academy of Sciences, 110(15), 6223. $<$ https://doi.org/10.1073/pnas.1219302110>

Arocena, Gustavo A. (2015a). Neurociencia, tratamiento penitenciario y reinserción social del condenado. Reflexiones en torno al Derecho Argentino. Revista General de Derecho Penal (23).

Arocena, Gustavo A. (2015b). El tratamiento penitenciario y los saberes neurocientíficos. Reflexiones desde el derecho argentino. Actualidad Penal (2015). Instituto Pacífico.

Asbury, C. H., Detre, J. A. (2019, julio). Brain Imaging Technologies: Clinical and Neuroscience Applications. Dana Fundation. < https://acortar.link/ TWEL9T>

Atmanspacher, H. (2014). Levels of unconsciousness and their formal structure. The Journal of Analytical Psychology 59(3), 385-390. PubMed. <https:// doi.org/10.1111/1468-5922.12087>

Beitz, C. R. (2011). The idea of human rights. Oxford University Press.

Bobbio, N. (2009). Sobre el fundamento de los derechos del hombre. En J. Binaghi (trad.), El problema de la guerra y las vías de la paz (2. ${ }^{\mathrm{a}} \mathrm{ed}$. , pp. 117-129). Gedisa.

Bonaci, T., Calo, R., Chizeck, H. J. (2014). App stores for the brain: Privacy $\&$ security in Brain-Computer Interfaces. 2014 IEEE International Symposium on Ethics in Science, Technology and Engineering, 1-7. $<$ https://doi. org/10.1109/ETHICS.2014.6893415>

Bublitz, J.-C. (2013). My Mind Is Mine!? Cognitive Liberty as a Legal Concept. En E. Hildt y A. G. Franke (eds.), Cognitive Enhancement: An Interdisciplinary Perspective (pp. 233-264). Springer Netherlands. $<$ https://doi. org/10.1007/978-94-007-6253-4_19>

Bunge, M. A. (1999). El problema de la mente-cerebro, un enfoque psicobiológico. Atalaya.

Burnett, S., Bault, N., Coricelli, G., Blakemore, S.-J. (2010). Adolescents' heightened risk-seeking in a probabilistic gambling task. Cognitive Development 25(2), 183-196. PubMed. <https://doi.org/10.1016/j.cogdev.2009.11.003> 
Burns, J. M., Swerdlow, R. H. (2003). Right Orbitofrontal Tumor With Pedophilia Symptom and Constructional Apraxia Sign. JAMA Neurology 60(3), 437-440. <https://doi.org/10.1001/archneur.60.3.437>

Cohen, A. O., Breiner, K., Steinberg, L., Bonnie, R. J., Scott, E. S., Taylor-Thompson, K., Rudolph, M. D., Chein, J., Richeson, J. A., Heller, A. S., Silverman, M. R., Dellarco, D. V., Fair, D. A., Galván, A., Casey, B. J. (2016). When Is an Adolescent an Adult? Assessing Cognitive Control in Emotional and Nonemotional Contexts. Psychological Science 27(4), 549562. <https://doi.org/10.1177/0956797615627625>

Dehaene, S. (2015). La conciencia en el cerebro. Descifrando el enigma de cómo el cerebro elabora nuestros pensamientos (M. J. D’Alessio, Trad.). Siglo XXI Editores.

Díaz, P. (2008). Implicancias de las técnicas de medición de la actividad cerebral en la cognición: ¿El tiempo o el espacio? Revista de Psicología 17(1). DOI: 10.5354/0719-0581.2011.17143. < https://revistapsicologia.uchile.cl/ index.php/RDP/article/view/17143>

Eklund, A., Nichols, T. E., Knutsson, H. (2016). Cluster failure: Why fMRI inferences for spatial extent have inflated false-positive rates. Proceedings of the National Academy of Sciences 113(28), 7900. <https://doi.org/10.1073/ pnas.1602413113>

Ferrajoli, L. (2016). Principia Iuris. Teoría del derecho y de la democracia (A. Ruiz Miguel, L. P. Sanchís, M. Gascón, J. C. Bayón Mohino, P. A. Ibáñez, trads.; 2. ${ }^{\text {e ed.). Trotta. }}$

García-López, E., Mercurio, E., Nijdam-Jones, A., Morales, L. A., Rosenfeld, B. (2019). Neurolaw in Latin America: Current Status and Challenges. International Journal of Forensic Mental Health 18(3), 260-280. $<$ https:// doi.org/10.1080/14999013.2018.1552634>

Gibson, W. G., Farnell, L., Bennett, M. R. (2007). A computational model relating changes in cerebral blood volume to synaptic activity in neurons. Neurocomputing 70(10-12), 1674-1679. <https://doi.org/10.1016/j. neucom.2006.10.071> 
Giedd, J. N. (2008). The Teen Brain: Insights from Neuroimaging. Journal of Adolescent Health 42(4), 335-343. <https://doi.org/10.1016/j. jadohealth.2008.01.007>

Giménez Amaya, J. M., Murillo, J. I. (2007). Mente y cerebro en la Neurociencia contemporánea. Una aproximación a su estudio interdisciplinar. Scripta Theologica 39(2), 607-635.

Greely, H. T. (2012). Direct Brain Interventions to "Treat" Disfavored Human Behaviors: Ethical and Social Issues. Clinical Pharmacology \& Therapeutics 91(2), 163-165. <https://doi.org/10.1038/clpt.2011.292>

Gutiérrez Martínez, J., Cantillo Negrete, J., Cariño Escobar, R. I., Elías Viñas, D. (2013). Los sistemas de interfaz cerebro-computadora: Una herramienta para apoyar la rehabilitación de pacientes con discapacidad motora. Investigación en Discapacidad 2(2), 62-69.

Harenski, C. L., Hamann, S. (2006). Neural correlates of regulating negative emotions related to moral violations. NeuroImage 30(1), 313-324. <https:// doi.org/10.1016/j.neuroimage.2005.09.034>

Hoaken, P. N. S., Allaby, D. B., Earle, J. (2007). Executive cognitive functioning and the recognition of facial expressions of emotion in incarcerated violent offenders, non-violent offenders, and controls. Aggressive Behavior 33(5), 412-421. <https://doi.org/10.1002/ab.20194>

Ienca, M., Andorno, R. (2017). Towards new human rights in the age of neuroscience and neurotechnology. Life Sciences, Society and Policy 13(1), 5. $<$ https://doi.org/10.1186/s40504-017-0050-1>

Ienca, M., Haselager, P. (2016). Hacking the brain: Brain-computer interfacing technology and the ethics of neurosecurity. Ethics and Information Technology 18(2), 117-129. <https://doi.org/10.1007/s10676-016-9398-9>

Lefaucheur, J.-P., André-Obadia, N., Antal, A., Ayache, S. S., Baeken, C., Benninger, D. H., Cantello, R. M., Cincotta, M., de Carvalho, M., De Ridder, D., Devanne, H., Di Lazzaro, V., Filipović, S. R., Hummel, F. C., Jääskeläinen, S. K., Kimiskidis, V. K., Koch, G., Langguth, B., Nyffeler, T., ... Garcia-Larrea, L. (2014). Evidence-based guidelines on the therapeutic use of repetitive transcranial magnetic stimulation (rTMS). Clinical Neurophysiology 125(11), 2150-2206. <https://doi.org/10.1016/j.clinph.2014.05.021> 
Levitskaya, O., Lebedev, M. (2016). Brain-computer interface: The future in the present. Bulletin of Russian State Medical University, 4-15. $<$ https:// doi.org/10.24075/brsmu.2016-02-01>

Lezaún, N. U. (1988). El problema mente-cerebro desde un enfoque evolucionista-emergentista: Una explicación conjetural. Revista internacional de los estudios vascos, RIEV 33(2), 187-222.

Libet, B. (1999). Do we have free will? Journal of Consciousness Studies 6(8-9), 47-57.

Ligthart, S. L. T. J. (2019). Coercive neuroimaging, criminal law, and privacy: A European perspective. Journal of Law and the Biosciences 6(1), 289-309. $<$ https://doi.org/10.1093/jlb/lsz015>

Loftus, E. F., Davies, G. M. (1984). Distortions in the memory of children. Journal of Social Issues 40(2), 51-67. <https://doi.org/10.1111/j.1540-4560.1984. tb01093.x>

Logothetis, N. K. (2008). What we can do and what we cannot do with fMRI. Nature 453, 869.

Lukomski, A. (2007). El problema mente-cuerpo. Logos. Anales Del Seminario de Metafísica [Universidad Complutense de Madrid, España] (12), 57-68.

Martínez Garay, L. (2020). Peligrosidad, Algoritmos y Due Process: El caso States vs. Loomis. Revista de Derecho Penal y Criminología (485). <https:// doi.org/10.5944/rdpc.20.2018.26484>

Martínez, S. M., Laino, N., Mariezcurrena, J. (2013). Justicia Penal Juvenil y Derecho al Recurso. Revista Derechos Humanos (Infojus) (4), 217-238.

Mendez, M. F. (2006). What frontotemporal dementia reveals about the neurobiological basis of morality. Medical Hypotheses 67(2), 411-418. $<$ https:// doi.org/10.1016/j.mehy.2006.01.048>

Mercurio, E. N. (2012). Cerebro y adolescencia: Implicancias jurídico-penales [Brain and adolescence: legal-criminal implications] (1. ${ }^{\mathrm{a}}$ ed.). Ad-Hoc.

Mercurio, E. N. (2014). Influencia de los avances en neurociencia en las decisiones judiciales en el derecho penal juvenil [Influence of advances in neuroscience in judicial decisions in juvenile criminal law]. En Informes en Derecho. Estudios de Derecho Penal Juvenil V. Defensoría Penal Pública. 
Mercurio, E. N., García López, E., Morales Quintero, L. A. (2019). Psicopatología forense y neurociencias: Aportaciones al sistema de justicia para adolescentes [Forensic Phsychopathology and Neuroscience: Contributions to the Justice System for Adolescents]. Boletín Mexicano de Derecho Comparado 1(153), 931-971.

Merkel, R. (2013). Nuevas intervenciones en el cerebro. Mejora de la condición mental humana y límites del derecho penal. En E. Demetrio Crespo, M. Maroto Calatayud (eds.), Neurociencias y Derecho Penal: Nuevas perspectivas en el ámbito de la culpabilidad y el tratamiento jurídico-penal de la

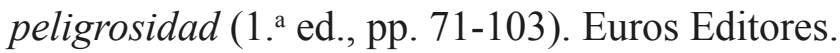

Miró-Llinares, F. (2018). Inteligencia Artificial y Justicia Penal: Más allá de los resultados lesivos causados por robots. Revista de Derecho Penal y Criminología (20), 87-130. <https://doi.org/10.5944/rdpc.20.2018.26446>

Moll, J., De Oliveira-Souza, R. (2007). Moral judgments, emotions and the utilitarian brain. Trends in Cognitive Sciences 11(8), 319-321. $<$ https://doi. org/10.1016/j.tics.2007.06.001>

Moll, J., Zahn, R., De Oliveira-Souza, R., Krueger, F., Grafman, J. (2005). The neural basis of human moral cognition. Nature Reviews Neuroscience 6(10), 799-809. <https://doi.org/10.1038/nrn1768>

Muñoz Ortega, J. M. (2013). Neurofilosofía y libre albedrío [Neurophilosophy and free will]. Daimon Revista Internacional de Filosofia (59), 57-70.

Muñoz Ortega, J. M. (2015). Mecánica Cuántica y Libre Albedrío: Cinco Cuestiones Fundamentales [Quantum Mechanics and Free Will: Five Fundamental Issues]. Principia: an international journal of epistemology 19(1), 65-92. <https://doi.org/10.5007/1808-1711.2015v19n1p65>

Muñoz Ortega, J. M. (2016). Libre Albedrío y Responsabilidad Moral: Una aproximación desde la Neurociencia [Free Will and Moral Responsibility: An Approach from Neuroscience]. Tesis doctoral, Universidad Nacional de Educación a Distancia, Facultad de Filosofía. <http://e-spacio.uned.es/ fez/eserv/tesisuned:Filosofia-Jmmunoz/MUNOZ_ORTEGA_Tesis.pdf $>$

Nickel, J. (2014). Human Rights. The Stanford Encyclopedia of Philosophy. $<$ https://plato.stanford.edu/archives/sum2019/entries/rights-human/> 
Normann, C., Berger, M. (2008). Neuroenhancement: Status quo and perspectives. European Archives of Psychiatry and Clinical Neuroscience 258(S5), 110-114. <https://doi.org/10.1007/s00406-008-5022-2>

Pardo, M. S., Patterson, D. (2011). Fundamentos filosóficos del Derecho y la neurociencia [Philosophical Fundations of Law and Neuroscience] (I. C. Vila, M. G. Bel, trads.). InDret: Revista para el Análisis del Derecho 2(819), $1-51$.

Pérez Manzano, M. (2011). Fundamento y fines del Derecho penal. Una revisión a la luz de las aportaciones de la neurociencia. InDret: Revista para el Análisis del Derecho (2), 1-40.

Pliszka, S. R., Glahn, D. C., Semrud-Clikeman, M., Franklin, C., Perez III, R., Xiong, J., Liotti, Mario (2006). Neuroimaging of Inhibitory Control Areas in Children With Attention Deficit Hyperactivity Disorder Who Were Treatment Naive or in Long-Term Treatment. American Journal of Psychiatry 163(6), 1052-1060. <https://doi.org/10.1176/ajp.2006.163.6.1052>

Poldrack, R. A., Monahan, J., Imrey, P. B., Reyna, V., Raichle, M. E., Faigman, D., Buckholtz, J. W. (2018). Predicting Violent Behavior: What Can Neuroscience Add? Trends in Cognitive Sciences 22(2), 111-123. <https:// doi.org/10.1016/j.tics.2017.11.003>

Rasche, D., Rinaldi, P. C., Young, R. F., Tronnier, V. M. (2006). Deep brain stimulation for the treatment of various chronic pain syndromes. Neurosurgical Focus FOC 21(6), 1-8. <https://doi.org/10.3171/foc.2006.21.6.10>

Roskies, A. L. (2008). Neuroimaging and Inferential Distance. Neuroethics 1(1), 19-30. <https://doi.org/10.1007/s12152-007-9003-3>

Sanguineti, J. J. (2005). Operazioni cognitive: Un approccio ontologico al problema mente cervello. Acta Philosophica 14(2), 233-258.

Schulz, K. P., Fan, J., Tang, C. Y., Newcorn, J. H., Buchsbaum, M. S., Cheung, A. M., Halperin, J. M. (2004). Response Inhibition in Adolescents Diagnosed With Attention Deficit Hyperactivity Disorder During Childhood: An Event-Related fMRI Study. American Journal of Psychiatry 161(9), 1650-1657. <https://doi.org/10.1176/appi.ajp.161.9.1650>

Shen, F. (2013). Neuroscience, Mental Privacy, and the Law. Harvard Journal of Law \& Public Policy (36). 
Sommaggio, P., Mazzocca, M. (2020). Cognitive Liberty and Human Rights. En A. D'Aloia, M. C. Errigo (eds.), Neuroscience and Law (pp. 95-111). Springer International Publishing. $<$ https://doi.org/10.1007/978-3-03038840-9_6>

Tronnier, V. M., Rasche, D. (2014). Deep Brain Stimulation. En D. Rasche, H. Knotkova (eds.), Textbook of Neuromodulation. Springer. <https://doi. org/10.1007/978-1-4939-1408-1_6>

Yuan, B. J. C., Hsieh, C. H., Chang, C. C. (2010). National technology foresight research: A literature review from 1984 to 2005. International Journal of Foresight and Innovation Policy 6(1/2/3), 5. <https://doi.org/10.1504/ IJFIP.2010.032663>

Yuste, R., Goering, S. (2016). On the Necessity of Ethical Guidelines for Novel Neurotechnologies. Cell 167(4), 882-885. $<$ https://doi.org/10.1016/j. cell.2016.10.029>

Yuste, R., Goering, S., Arcas, B. A., Bi, G., Carmena, J. M., Carter, A., Fins, J. J., Friesen, P., Gallant, J., Huggins, J. E., Illes, J., Kellmeyer, P., Klein, E., Marblestone, A., Mitchell, C., Parens, E., Pham, M., Rubel, A., Sadato, N., ... Wolpaw, J. (2017). Four ethical priorities for neurotechnologies and AI. Nature 551(7679), 159-163. <https://doi.org/10.1038/551159a >

Zaffaroni, E. R. (2012). Crímenes de masa (2. ${ }^{\mathrm{a}}$ ed.). Ediciones Madres de Plaza de Mayo. 